This is a post-print version of the following article: Degradation of selected agrochemicals by the white rot fungus Trametes versicolor.

DOI: 10.1016/j.scitotenv.2014.08.116

\title{
Degradation of selected agrochemicals by the white rot fungus Trametes versicolor
}

\author{
Josep Anton Mir-Tutusaus ${ }^{\mathrm{a}}$, Mario Masís-Mora ${ }^{\mathrm{b}}$, CayoCorcellas ${ }^{\mathrm{c}}$, Ethel Eljarrat ${ }^{\mathrm{c}}$, Damià \\ Barceló $^{\mathrm{c}, \mathrm{d}}$, Montserrat Sarràa , Glòria Caminale ${ }^{\mathrm{e}}$, Teresa Vicent ${ }^{\mathrm{a}}$, Carlos E. Rodríguez- \\ Rodríguez ${ }^{\mathrm{a}, \mathrm{b}, *}$ \\ a Departa ment d'Enginyeria Química, Escola d'Enginyeria, Universitat A utònoma de Barcelona, 08193 \\ Bellaterra, Barcelona, Spain \\ ${ }^{\mathrm{b}}$ Centro de Investigación en Contaminación Ambiental (CICA), Universidad de Costa Rica, 2060 San \\ José, Costa Rica \\ ${ }^{c}$ Depart ment of Environmental Chemistry, Institute of Environ mental Assessment and Water Research \\ (IDAEA), Spanish Council for Scientific Research (CSIC), Jord i Girona 18-26, E-08034 Barcelona, \\ Spain \\ ${ }^{\mathrm{d}}$ Catalan Institute forWater Research (ICRA), H2O Build ing, Scientific and Technological Park of the \\ University of Girona, Emili Grahit 101, 17003 Girona, Spain \\ ${ }^{\text {e }}$ Institut de Química Avançada de Catalunya (IQAC) CSIC. Jordi Girona 18-26, 08034 Barcelona, Spain
}

\section{Abstract}

Use of agrochemicals is a worldwide practice that exerts an important effect on the environment; therefore the search of approaches for the elimination of such pollutants should be encouraged. The degradation of the insecticides imiprothrin (IP) and cypermethrin (CP), the insecticide/nematicide carbofuran (CBF) and the antibiotic of agricultural use oxytetracycline (OTC) were assayed with the white rot fungus Trametes versicolor. Experiments with fungal pellets demonstrated extensive degradation of the four tested agrochemicals, at rates that followed the pattern IP > OTC > CP > CBF. In vitro assays with laccase-mediator systems showed that this extracellular enzyme participates in the transformation of IP but not in the cases of CBF and OTC. On the other hand, in vivo studies with inhibitors of cytochrome $\mathrm{P} 450$ revealed that this intracellular system plays an important role in the degradation of IP, OTC and CBF, but not for CP. The compounds 3-(2,2-dichlorovinyl)-2,2-dimethylcyclopropane carboxylic acid (DCCA) and 3-phenoxybenzoic acid (PBA) were detected as transformation products of $\mathrm{CP}$, as a result of the breakdown of the molec ule. Meanwhile, 3hydroxycarbofuran was detected as a transformation product of CBF; this metabolite tended to accumulate during the process, nonetheless, the toxicity of the system was effectively reduced. Simultaneous degradation of $\mathrm{CBF}$ and OTC showed a reduction in toxicity; similarly, when successive additions of OTC were done during the slower degradation of $\mathrm{CBF}$, the fungal pellets were able to degrade both compounds. The 
simultaneous degradation of the four compounds successfully took place with minimal inhibition of fungal activity and resulted in the reduction of the global toxicity, thus supporting the potential use of $T$. versicolor for the treatment of diverse agrochemicals.

\section{Highlights}

- Trametes versicolor efficiently degrades four different agrochemicals simultaneously.

- Order of degradation rates is:

imiprothrin > oxytetracycline > cypermethrin > carbofuran.

-Degradation process usually decreases the initial toxicity of the agrochemicals.

- Cytochrome P450 is involved in the degradation, except for cypermethrin.

-Laccase plays a role in the transformation of imiprothrin.

Keywords

Agrochemicals; Pesticides; Antibiotics; Degradation; Fungi

\section{Introduction}

The term agrochemical is given to various chemical products widely used in agriculture as prophylactic treatment to maintain high agricultural production (Sequinatto, 2013). Among the organic pollutants, agrochemicals stand out as their application in most cases is linked to direct contact with the environment, which results in high risk of exposure to living beings. Many agrochemicals may affect non-target organisms and contaminate soil, water sources and air.

Insecticides are widely used in pest control in households as well as in agricultural systems. In particular, pyrethroids account for $25 \%$ of the worldwide insecticide market (Zhang et al., 2010). With the restriction on the use of organophosphates and carbamates, pyrethroids like imiprothrin (IP) and cypermethrin (CP) have generally been regarded as the replacements, because they have higher insect-to-mammalian toxicity ratio (Hintzen et al., 2009). Despite being less harmful to humans than organophosphates and carbamates, there is an increasing concern as they present reproductive and neurological toxicity, endocrine disruptive effects, and high acute toxicity to fish and aquatic invertebrates (Abdallah et al., 2010 and Shafer et al., 2005). In addition, long-term exposure to pyrehtroids may lead to chronic diseases (Aksakal et al., 2010) and some of them are considered as possible human carcinogens by the Environmental Protection Agency (EPA).

Carbofuran $(\mathrm{CBF})$ is an agrochemical mainly employed as an insecticide/nematicide. $\mathrm{CBF}$ belongs to the family of carbamates, pesticides that act through the inhibition of acetylcholinesterase, an enzyme that degrades the neurotransmitter acetylcholine in 
synapses from vertebrates and invertebrates. It is highly toxic to mammals and reportedly embryotoxic and teratogenic (Gupta, 1994). CBF has been banned in agricultural practice in the United States and the European Union, but it is still widely used in other latitudes.

Oxytetracycline (OTC) is a broad-spectrum antibiotic included in the group of tetracyclines. OTC interferes in bacterial protein synthesis. It is one of only two antimicrobials (as streptomycin) to be registered by the EPA for use in plant agriculture as a prophylactic treatment (Vidaver, 2002). Environmental risk of release of antibiotics is related to the growing resistance of pathogenic bacteria and the potential dissemination of antibiotic resistance, which is considered as an ecological problem (Kümmerer, 2004). Moreover, antibiotics have the potential to affect microbial communities and therefore unbalance natural processes that take place in the environment like organic matter degradation (Kümmerer, 2009).

Besides reducing the use of these pesticides and finding a less harmful replacement, polluted sites must be decontaminated. Therefore, remediation techniques must be developed and implemented to remove theses residues from the environment. White rot fungi (WRF) have the potential to transform and sometimes mineralize a broad spectrum of xenobiotics such as textile dyes (Borchert and Libra, 2001 and Swamy and Ramsay, 1999), chlorinated solvents (Mendoza-Cantu et al., 2000), polycyclic aromatic hydrocarbons (PAHs) (Canet et al., 2001 and Zheng and Obbard, 2001), brominated flame retardants (Zhou et al., 2007), pharmaceuticals (Hata et al., 2010 and MarcoUrrea et al., 2009) and UV filters (Gago-Ferrero et al., 2012). They use a nonspecific enzymatic system that includes extracellular lignin modifying enzymes (mainly laccase, lignin peroxidase, manganese peroxidase and versatile peroxidase) and intracellular enzymes such as the cytochrome P450 system, among others (Asgher et al., 2008).

The purpose of the present study was to evaluate the capacity of the WRF Trametes versicolor to degrade selected agrochemicals with different structures and action mechanisms. Toxicological aspects were considered to assay the pertinence of the process, and transformation products were detected for $\mathrm{CBF}$ and $\mathrm{CP}$.

\section{Materials and methods}

\subsection{Chemicals and fungal strain}

CBF (98\%), OTC (> 95\%), IP (33442, PESTANAL®) and CP (36128, PESTANAL®) analytical standards were purchased from Sigma-Aldrich (Barcelona, Spain). For degradation experiments commercial insecticide Baygon® (imiprothrin $0.1 \mathrm{~g} / 100 \mathrm{~g}$, cypermethrin $0.1 \mathrm{~g} / 100 \mathrm{~g}$; Johnson's Wax Spain, Madrid) was used. Purified laccase (> $20 \mathrm{U} \mathrm{mg}^{-1}$ ) from T. versicolor was acquired from Sigma Aldrich (Barcelona, Spain). Malt extract was purchased from Scharlau (Barcelona, Spain). The laccase mediator 2,2-azino-bis-(3- ethylbenzthiazoline-6-sulfonic acid) diammonium salt (ABTS) (> 98\%) and the cytochrome P450 inhibitor 1-aminobenzotriazole (ABT) (> 98\%) were 
purchased from Sigma-Aldrich (Barcelona, Spain). Laccase mediators 1hydroxybenzotriazole hydrate (HOBt) (> 97\%) and violuric acid (VA) (> 97\%) were acquired from Fluka (Barcelona, Spain). Other chemicals were of the highest available purity and were purchased from Sigma-Aldrich (Barcelona, Spain).

T. versicolor (ATCC\#42530) was maintained by subculturing on $2 \%$ malt extract agar slants (pH 4.5) at $25{ }^{\circ} \mathrm{C}$. Subcultures were routinely made every $30 \mathrm{~d}$. A mycelial suspension was prepared as described elsewhere (Font et al., 2003). Pellets of $T$. versicolor were produced by inoculating $1 \mathrm{~mL}$ of this mycelial suspension in a $1 \mathrm{~L}$ Erlenmeyer flask containing $250 \mathrm{~mL}$ of malt extract broth $\left(20 \mathrm{~g} \mathrm{~L}^{-1}\right)$ with shaking $(130 \mathrm{rpm}$, radius $=25 \mathrm{~mm})$ at $25{ }^{\circ} \mathrm{C}$ for $5 \mathrm{~d}$.

For the analytical determination of pyrethroid metabolites 3-(2,2-dichlorovinyl)-2,2dimethylcyclopropane carboxylic acid (DCCA), $\mathrm{d}_{6}$-cis-DCCA and 3-phenoxybenzoic acid (PBA) were purchased from Dr. Ehrenstorfer (Augsburg, Germany). ${ }^{13} \mathrm{C}$-PBA was purchased from Cambridge Isotope Laboratories, Inc. (Andover, MA, USA). Solvents with quality for organic residue analysis were purchased from J.T. Baker (Deventer, The Netherlands). For the determination of transformation products from CBF, 3hydroxycarbofuran $(99.5 \%)$ and 3-ketocarbofuran $(99.5 \%)$ were obtained from Chemservice (West Chester, Pennsylvania, USA).

\subsection{Experimental procedures}

\subsubsection{In vivo degradation experiments}

All in vivo experiments were carried out using T. versicolor pellets. Degradation experiments for IP and $\mathrm{CP}$ were performed in $125 \mathrm{~mL}$ amber bottles containing $0.8 \mathrm{~g}$ of wet mycelial pellets (equivalent to $0.03 \mathrm{~g} \mathrm{DW}$ ) in a total volume of $10 \mathrm{~mL}$ of a chemically defined medium. Defined medium contained per liter: 8 g glucose, $0.05 \mathrm{~g}$ ammonium tartrate, 10 and $100 \mathrm{~mL}$ respectively, of a micro and macronutrient solution (Kirk et al., 1978) and $1.168 \mathrm{~g}$ 2,2-dimethylsuccinate. Each unitary experiment was conducted in triplicate and included uninoculated controls and heat-killed controls (autoclaved cultures cultivated under identical conditions to those of experimental cultures) to evaluate adsorption. Commercial IP and CP emulsion mixture was added into the bottles at a final concentration of $10 \mathrm{mg} \mathrm{L}^{-1}$ each. After insecticides addition, amber bottles were incubated under shaken conditions $(130 \mathrm{rpm})$ at $25^{\circ} \mathrm{C}$ in the dark, to obviate the possible influence of light on CP and IP stabilities. At each sample point unitary triplicates were sacrificed and extracted as described in Section 2.3.1, and analyzed as in Section 2.3.2. Experiments for degradation of CBF and OTC were done in $250 \mathrm{~mL}$ flasks containing $10 \mathrm{~g}$ wet fungal pellets in a total volume of $100 \mathrm{~mL}$ defined medium. Samples were withdrawn from triplicate cultures at each sampling point and included analogous controls as described above. When indicated, a final concentration of $5 \mathrm{mM}$ of ABT, the cytochrome P450 inhibitor, was added. Abiotic degradation of agrochemicals in time-course experiments was calculated by comparing the initial and final concentrations of the uninoculated controls; ad sorption of the pesticides was 
determined from the difference in the agrochemical concentrations between uninoculated and heat-killed controls; minimum biodegradation is the difference between the final concentration of pesticides of the experimental flasks and that of the heat-killed control; total elimination is the sum of abiotic degradation, adsorption and minimum biodegradation.

\subsubsection{Assays with laccase and laccase-mediator systems}

Laccase and laccase-mediator degradation experiments were performed per triplicate in $250 \mathrm{~mL}$ Erlenmeyer flasks containing $100 \mathrm{~mL}$ of a purified laccase solution at $1000 \mathrm{U}$ $\mathrm{L}^{-1}$ ( $\mathrm{pH} 4.5$ ). Effect of laccase mediators was evaluated by the addition of HOBt, ABTS and VA at a final concentration of $1 \mathrm{mM}$. All flasks contained $10 \mathrm{mg} \mathrm{L}^{-1}$ of CBF, OTC or the IP and CP mixture and were shaken $(130 \mathrm{rpm})$ at $25^{\circ} \mathrm{C}$. For IP and CP, $5 \mathrm{~mL}$ samples were taken at $0,3,8$ and $24 \mathrm{~h}$, and extracted and analyzed as described in $\underline{2.3 .1}$ and 2.3.2, respectively. In experiments with CBF and OTC, $1 \mathrm{~mL}$ samples at designated times (from $0 \mathrm{~h}$ to $96 \mathrm{~h}$ ) were withdrawn, and $100 \mu \mathrm{L}$ acetic acid was added to stop the reaction (García-Galán et al., 2011 and Rodríguez-Rodríguez et al., 2012b) prior analysis as indicated in 2.3.2.

\subsection{Analytical procedures}

\subsubsection{Sample processing}

Extraction of IP and CP was performed in situ inside the experimental bottles. An equal volume of chloroform was added to the medium contained in the bottles $(1: 1 \mathrm{v} / \mathrm{v})$ and extracted by 5 min sonication at $30{ }^{\circ} \mathrm{C}$ in a sonication bath (Branson 3510 Ultrasonic Cleaner, Branson Ultrasonics Corporation, Danbury, US) and vigorously shaken manually for $1 \mathrm{~min}$. The emulsion was centrifuged for $2 \mathrm{~min}$ at $3500 \mathrm{rpm}$, and the organic phase was transferred to a vial. The chloroform was then evaporated under a nitrogen stream, and the concentrated extracts were reconstituted in $1 \mathrm{~mL}$ of methanol. The extraction yield was $63 \pm 10 \%$. Samples for CBF and OTC analyses were obtained by withdrawing $1.5 \mathrm{~mL}$ broth from the culture flasks, and subsequently centrifuging at $3000 \mathrm{rpm}$ for $5 \mathrm{~min}$; supernatant was directly injected in the HPLC; recoveries for OTC and $\mathrm{CBF}$ were $>90 \%$.

\subsubsection{Analysis of agrochemicals and transformation products of CP and CBF}

The pesticide extracts were filtered through a $0.45 \mu \mathrm{m}$ membrane and placed in amber HPLC vials to avoid photodegradation. The samples were analyzed on a Dionex Ultimate 3000 HPLC system equipped with a UV detector and a GraceSmart RP18 column $(250 \mathrm{~mm} \times 4.6 \mathrm{~mm}$, particle size $5 \mu \mathrm{m})$. Detection of IP and CP was done at $225 \mathrm{~nm}$. A ramp of acetonitrile (B) and MilliQ water (A) was used as the mobile phase at a flow rate of $1.5 \mathrm{~mL} \mathrm{~min}^{-1}$ : the eluent gradient started with $50 \% \mathrm{~B}$ and increased to $100 \% \mathrm{~B}$ from 0 to $20 \mathrm{~min}$; from 20 to $21 \mathrm{~min}$ the gradient decreased to $50 \% \mathrm{~B}$ and remained at $50 \%$ B for 4 more minutes. The injection volume was $40 \mu \mathrm{L}$, and the 
temperature of the column was maintained at $30{ }^{\circ} \mathrm{C}$. Detection of $\mathrm{CBF}$ was done at $215 \mathrm{~nm}$; chromatographic separation was achieved with a mobile phase of methanolwater (60:40), added isocratically at $1 \mathrm{~mL} \mathrm{~min}^{-1}$ (Plangklang and Reungsang, 2010). The injection volume was $10 \mu \mathrm{L}$, and column temperature was maintained at $30{ }^{\circ} \mathrm{C}$. Limit of detection for CBF was $0.2 \mathrm{mg} \mathrm{L}^{-1}$. Detection of OTC was performed at $355 \mathrm{~nm}$; separation of $10 \mu \mathrm{L}$ samples employed a mobile phase of methanolacetonitrile-oxalic acid $(0.01 \mathrm{M})(11: 22: 67)$ added isocraticaly at $1 \mathrm{~mL} \mathrm{~min}^{-1}$ at $30{ }^{\circ} \mathrm{C}$ (Yuan et al., 2011). Limit of detection for OTC was $0.2 \mathrm{mg} \mathrm{L}^{-1}$.

For the analyses of PBA and DCCA, metabolites of CP, an aliquot of the filtered extracts $(180 \mu \mathrm{L})$ was analyzed by previous addition of $20 \mu \mathrm{L}$ of an internal standard solution, consisting of ${ }^{13} \mathrm{C}$-PBA $\left(0.05 \mathrm{ng} \mu \mathrm{L}^{-1}\right)$ and $\mathrm{d}_{6}$-cis-DCCA $\left(0.1 \mathrm{ng} \mu \mathrm{L}^{-1}\right)$. Analyses were carried out with an LC system Symbiosis Pico (Spark Holland, Emmen, The Netherlands) using a Purospher® STAR RP-18 end-capped column (125 $\mathrm{mm} \times 2 \mathrm{~mm} \times 5 \mu \mathrm{m}$ particle size), supplied by Merck (Darmstadt, Germany) preceded by a C18 guard column $(2.1 \mathrm{~mm} \times 10 \mathrm{~mm})$ supplied by Waters. The mobile phases were acidified water $(0.1 \%$ acetic acid) and acetonitrile. The program worked isocratically with $50 \%$ of each solvent. Mass spectrometric analysis was performed with a hybrid QqLIT Applied Biosystem MSD Sciex 4000QTRAP (Applied Biosystems, Foster City, CA) using ESI mode. Experiments were carried out in negative ionization mode. Selected transitions for PBA were: $213 \rightarrow 169$ (quantification; declustering potential, DP - $45 \mathrm{~V}$; collision energy, CE - $20 \mathrm{~V}$; collision cell exit potential, CXP $-10 \mathrm{~V}$ ) and $213 \rightarrow 93$ (confirmation; DP $-40 \mathrm{~V}$; CE - $30 \mathrm{~V}$; CXP - $10 \mathrm{~V}$ ); selected transitions for DCCA were $207 \rightarrow 35$ (quantification; DP $-25 \mathrm{~V}$; CE $-30 \mathrm{~V}$; CXP $-5 \mathrm{~V}$ ) and $209 \rightarrow 35$ (confirmation; DP $-25 \mathrm{~V}$; CE $-20 \mathrm{~V}$; CXP $-10 \mathrm{~V}$ ), ‘following Olsson et al. (2004) ind ications. Other parameters were: entrance potential - $10 \mathrm{~V}$; temperature $550{ }^{\circ} \mathrm{C}$; ion source gas $50 \mathrm{psi}$; ionspray voltage $-4400 \mathrm{~V}$; curtain gas $10 \mathrm{psi}$; CAD gas medium. Limits of detection and quantification were $0.36 \mu \mathrm{g} \mathrm{L}^{-1}$ and $1.2 \mu \mathrm{g} \mathrm{L}^{-1}$, respectively for PBA, and $0.27 \mu \mathrm{g} \mathrm{L}^{-1}$ and $0.9 \mu \mathrm{g} \mathrm{L}^{-1}$, respectively for DCCA. Recoveries were $70 \%$ and $71 \%$ for PBA and DCCA, respectively.

Analyses of transformation products from CBF (3-hydroxycarbofuran and 3ketocarbofuran) were performed using an HPLC system (1200 series, Agilent Technologies, CA, USA) coupled to a 6130 quadrupole mass spectrometer with a G1978B multimode ion source (ESI and atmospheric pressure chemical ionization, APCI). Chromatographic separation was achieved with an Agilent Eclipse XDB-C18 column (150 mm $\times 4.6 \mathrm{~mm}$ i.d., particle size $5 \mu \mathrm{m}$; Agilent Technologies, CA, USA) and two mobile phases: water containing $0.1 \%$ formic acid (A) and methanol containing $0.1 \%$ formic acid (B). Gradient employed was as follows: $60 \%$ B for $3 \mathrm{~min}$, a $22 \mathrm{~min}$ linear gradient to $100 \% \mathrm{~B}, 5 \mathrm{~min}$ at $100 \% \mathrm{~B}$ and $0.1 \mathrm{~min}$ gradient back to $60 \% \mathrm{~B}$, followed by $5.9 \mathrm{~min}$ at initial conditions. The mobile phase flow rate was $0.4 \mathrm{~mL} \mathrm{~min}^{-1}$, and the injection volume was $25 \mu \mathrm{L}$. The mass spectrometer was operated in the selected-ion monitoring (SIM) positive mode electrospray ionization and was set up to monitor the ions $m / z 260$ and $m / z 163$ (3-hydroxycarbofuran), and $m / z 236$ and $\mathrm{m} / z, 179$ 
(3-ketocarbofuran). The mass spectrometry detector was set up using the following conditions: scanning range, $100-400$; drying gas $\left(\mathrm{N}_{2}\right)$ flow, $5 \mathrm{~L} / \mathrm{min}$; nebulizer pressure, $60 \mathrm{psi}$; dry gas temperature, $350{ }^{\circ} \mathrm{C}$; vaporizer temperature, $200{ }^{\circ} \mathrm{C}$; capillary voltage, $2000 \mathrm{~V}$; and fragmentor voltage, $90 \mathrm{~V}$. Recovery of the method was $75 \%$. Limit of detection and limit of quantification were $10 \mu \mathrm{g} \mathrm{L}^{-1}$ and $21 \mu \mathrm{g} \mathrm{L}^{-1}$ for CFN and 3-hydroxycarbofuran; $88 \mu \mathrm{g} \mathrm{L}^{-1}$ and $164 \mu \mathrm{g} \mathrm{L}^{-1}$ for 3-ketocarbofuran.

\subsubsection{Vibrio fischeri bioluminescence inhibition test (Microtox Test)}

A Microtox acute toxicity bioassay kit from Microbics was used in toxicity tests. $V$. fischeri is a marine bioluminescent bacterium that emits in the range of the visible light (maximum intensity at $490 \mathrm{~nm}$ ). Toxicity data were based on the decrease of bacterial bioluminescence after $5 \mathrm{~min}$ and $15 \mathrm{~min}$ of exposure to dilutions of the samples $(\mathrm{pH} 7)$ at $25^{\circ} \mathrm{C}$. Toxicity was expressed as toxicity units (TU). The experimental samples tested were collected from time-course degradation experiments described in

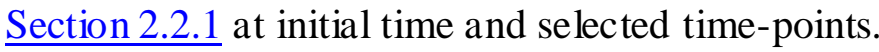

\subsubsection{Other analysis}

Laccase activity was measured using a modified version of the method for manganese peroxidase determination (Wariishi, 1992); the reaction mixture consisted of $200 \mu \mathrm{L}$ sodium malonate ( $250 \mathrm{mM}, \mathrm{pH} 4.5), 50 \mu \mathrm{L}$ 2,6-dimethoxyphenol (DMP, $20 \mathrm{mM}$ ) and $600 \mu \mathrm{L}$ sample. DMP is oxidized by laccase even in the absence of a cofactor. Changes in the absorbance at $468 \mathrm{~nm}$ were monitored for $2 \mathrm{~min}$ at $30{ }^{\circ} \mathrm{C}$. Results were expressed as $U$ per liter. One $U$ was defined as the number of micromoles of DMP oxidized per min. The DMP extinction coefficient was $24,800 \mathrm{M}^{-1} \mathrm{~cm}^{-1}$. Glucose concentration was measured with a biochemical analyzer YSI 2700 SELECT (Yellow Spring Instruments) in the concentration range $0-20 \pm 0.04 \mathrm{~g} \mathrm{~L}^{-1}$. Biomass amount (dry weight) was determined as the constant weight at $100{ }^{\circ} \mathrm{C}$.

\section{Results and discussion}

\subsection{Degradation of pyre throids (IP/CP)}

Time-course in vivo experiments with IP and $\mathrm{CP}$ at $10 \mathrm{mg} \mathrm{L}^{-1}$ showed a steep drop in concentration of IP but a less abrupt decrease in CP concentration (Fig. 1A And B, respectively) in experimental flasks. Elimination values were higher than $90 \%$, achieved after $2 \mathrm{~d}$ for IP and $15 \mathrm{~d}$ for CP. These differences in the degradation efficiencies could be dependent on the molecular structure of the pyrethroids, as it has been reported

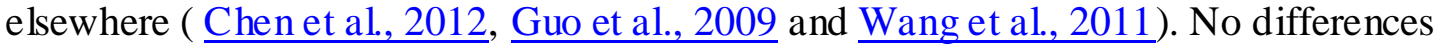
were found in pyrethroid concentrations at the end of the treatment between the uninoculated and the heat-killed controls, which indicates that the role of adsorption of the pollutants to biomass is negligible. Nonetheless, the concentrations of IP and CP were reduced by $19 \%$ and $23 \%$, respectively, finding that could be explained by abiotic losses. Minimum biodegradation was calculated by subtracting the abiotic removal 
value to the $90 \%$ degradation value, which resulted in $71 \%$ and $67 \%$ for IP and $\mathrm{CP}$, respectively. Separate quantification of CP isomers (cis-CP and trans-CP) revealed that the fungal removal of $\mathrm{CP}$ was not dependent on geometric isomeris m (data not shown).
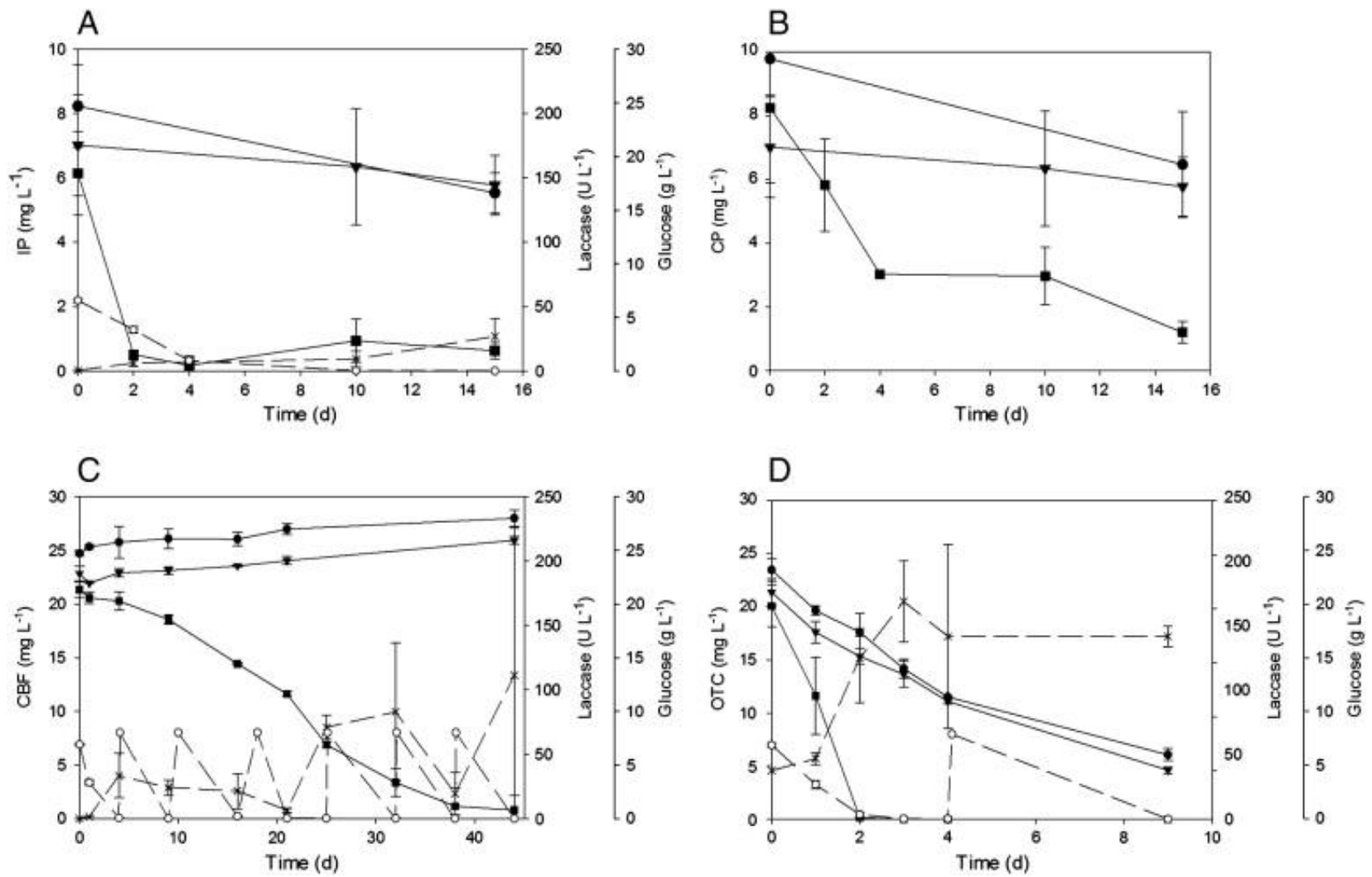

Fig. 1. Degradation profiles of IP (A), CP (B), CBF (C) and OTC (D) by T. versicolor. Sy mbols: uninoculated controls $(\bullet)$, heat-killed controls $(\boldsymbol{\nabla})$, reaction cultures $(\boldsymbol{\bullet})$, glucose $(\circ)$ and laccase activity $(\times)$. Values plotted are the means \pm standard deviation (SD) for triplicate cultures. Laccase and glucose consumption profiles in CP are the same as in IP. Values plotted are the means \pm SD for trip licate cultures.

Degradation of CP was confirmed by the detection of two transformation products (Fig. 2) which appear as a result of the breakdown of the molecule: DCCA and PBA. The molar amount of DCCA accumulated at the end of the assay in the heat-killed and abiotic controls approximately corresponds to the molar losses observed for CP (stoichiometry of the hydrolysis reaction is 1:1); however further DCCA elimination by the fungus is suggested, as the molar amount of this transformation product at time $15 \mathrm{~d}$ was lower than the molar reduction in CP during the same period. In the case of PBA, detected concentrations were very low compared to those of DCCA (1000-fold) even in the controls, suggesting high instability of the product in the matrix and making it difficult to conclude whether PBA is further eliminated by biological action. Microbial degradation of pyrethroids has been mostly reported for bacterial strains. Nonetheless, a fungal strain from the genus Cladosporium isolated from sludge, showed the capacity to remove several pyrethroids, including CP at efficiencies over $90 \%$ in only $5 \mathrm{~d}$ in liquid

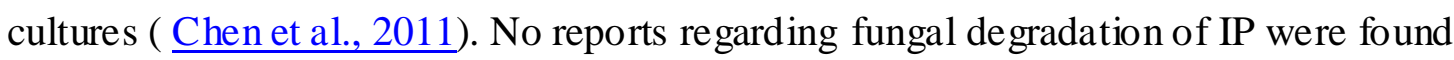
in specialized literature. 


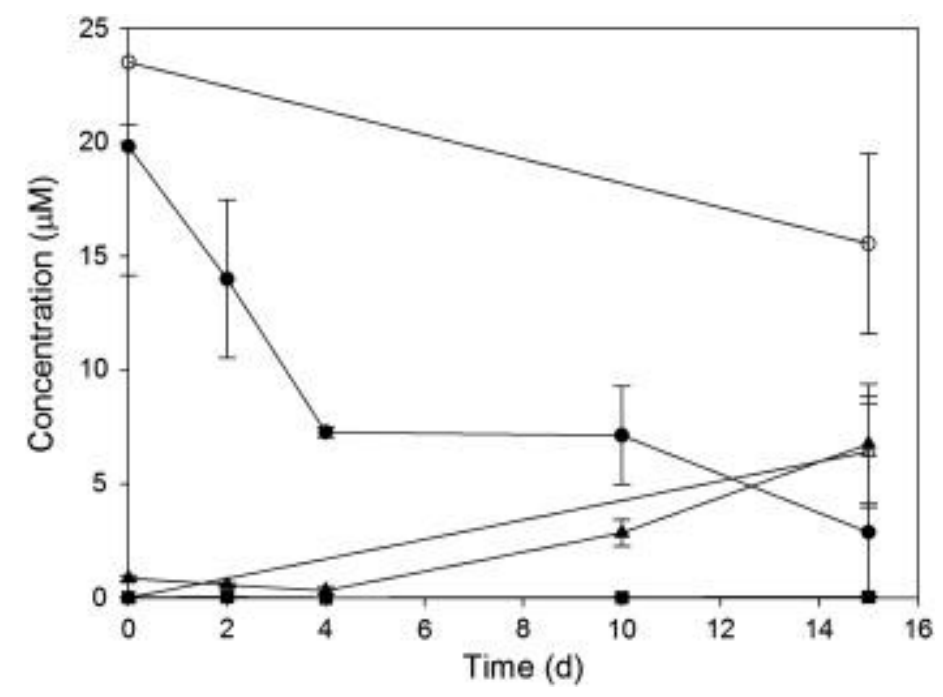

Fig. 2. Time-course evolution of major transformation products in the degradation of CP by $T$. versicolor pellets. Symbols: $(\bullet) \mathrm{CP} ;(\boldsymbol{\bullet}) \mathrm{PBA} ;(\boldsymbol{\Delta})$ DCCA. Filled symbols correspond to inoculated experiments and empty symbols to uninoculated ones. Values plotted are the means \pm SD for trip licate cultures.

A continuous increase in extracellular laccase activity was detected throughout the experiment (Fig. 1), from the day 10 onwards. As laccase was constitutively produced in the cultures, studies were carried out to define the involvement of laccase and laccase- mediator systems in the degradation of IP and CP. Laccases are multicoppercontaining oxidases that catalyze the oxidation of substituted phenols, anilines and aromatic thiols (Cañas and Camarero, 2010). Laccase mediator ABTS can oxidize nonphenolic compounds via an electron transfer route described elsewhere (Baiocco et al., 2003). The other mediators, HOBt and violuric acid, generate a highly reactive nitroxyl radical (> N-O) when oxidized by laccase, which subsequently oxidizes the target substrate by a mechanism of hydrogen atom trans fer (Fabbrini et al., 2002). Enzymatic assays with purified laccase at $1000 \mathrm{U} \mathrm{L}^{-1}$ failed to transform IP and CP. In contrast, when any of the mediators were added to the mixture, $70-100 \%$ of the original IP was transformed after $8 \mathrm{~h}$ and $>90 \%$ after $24 \mathrm{~h}$ (Fig. 3). However, cypermethrin showed a different behavior, and negligible transformation was achieved with the addition of HOBt or VA. Although these mediators are synthetic and are not found in the nature, some lignin-related phenols and unsaturated fatty acids present in the fungal mycelium can act as mediators and promote oxidation of pollutants in the environment ( $\underline{\text { Cañas and }}$ Camarero, 2010). Thus, the results suggest that laccase plays a role on the degradation of IP, whereas this role is not clear in the case of CP. Together with laccase, cytochrome $\mathrm{P} 450$ system has proved important in the detoxification of some environmental contaminants, and it is present not only in WRF but in bacteria as well (Doddapaneni and Yadav, 2004 and Lewis and Wiseman, 2005). Its role in the transformation of IP and $\mathrm{CP}$ was asses sed by the addition of the cytochrome $\mathrm{P} 450$ inhibitor ABT in $T$. versicolor cultures. In the case of IP ( Fig. 4A) the addition of the inhibitor induced a delay in its biotransformation in comparison with the inhibitor-free flasks. Degradation of $\mathrm{CP}$ occurred at the same rate in inhibited and inhibitor-free bottles, as the differences were not statistically significant $(\mathrm{p}=0.05)(\underline{\text { Fig. 4B }})$. The results, therefore, suggest that 
both laccase and the cytochrome P450 are involved in the transformation of IP; meanwhile the cytochrome $\mathrm{P} 450$ seems not involved in CP degradation. On the other hand, the slow but steady decrease of CP concentration in the laccase/med iators experiments suggests that laccase could play a role in $\mathrm{CP}$ degradation when used together with mediators, but other enzymatic compounds are likely to act in the process. In this respect, carboxylesterases are regarded as the main enzymes linked to pyrethroid degradation in pyrethroid-degrading bacteria, where ester hydrolysis of the carboxylester linkage is the main degradation pathway ( Guo et al., 2009 and Wang et al., 2011). Similarly, yeasts show pyrethroid hydrolyzing enzymes, and may have broad-spectrum substrate specificity (Chen et al., 2012).
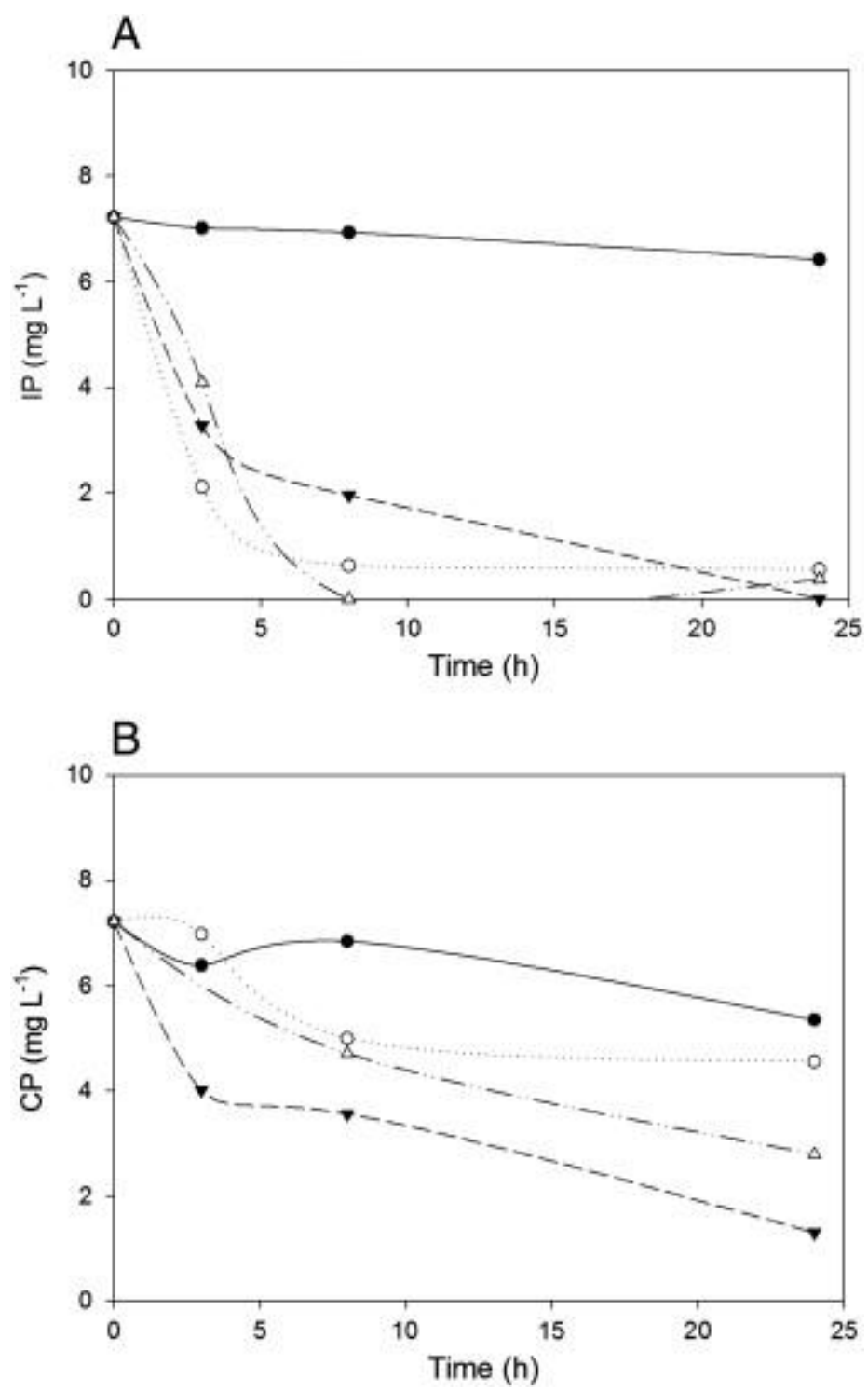

Fig. 3. Degradation profiles of IP (A) and CP (B) by purified laccase. Sy mbols: laccase without med iators $(\bullet)$, laccase/HOBt $(\circ)$, laccase/ABTS $(\boldsymbol{\nabla})$ and laccase/VA $(\Delta)$. 

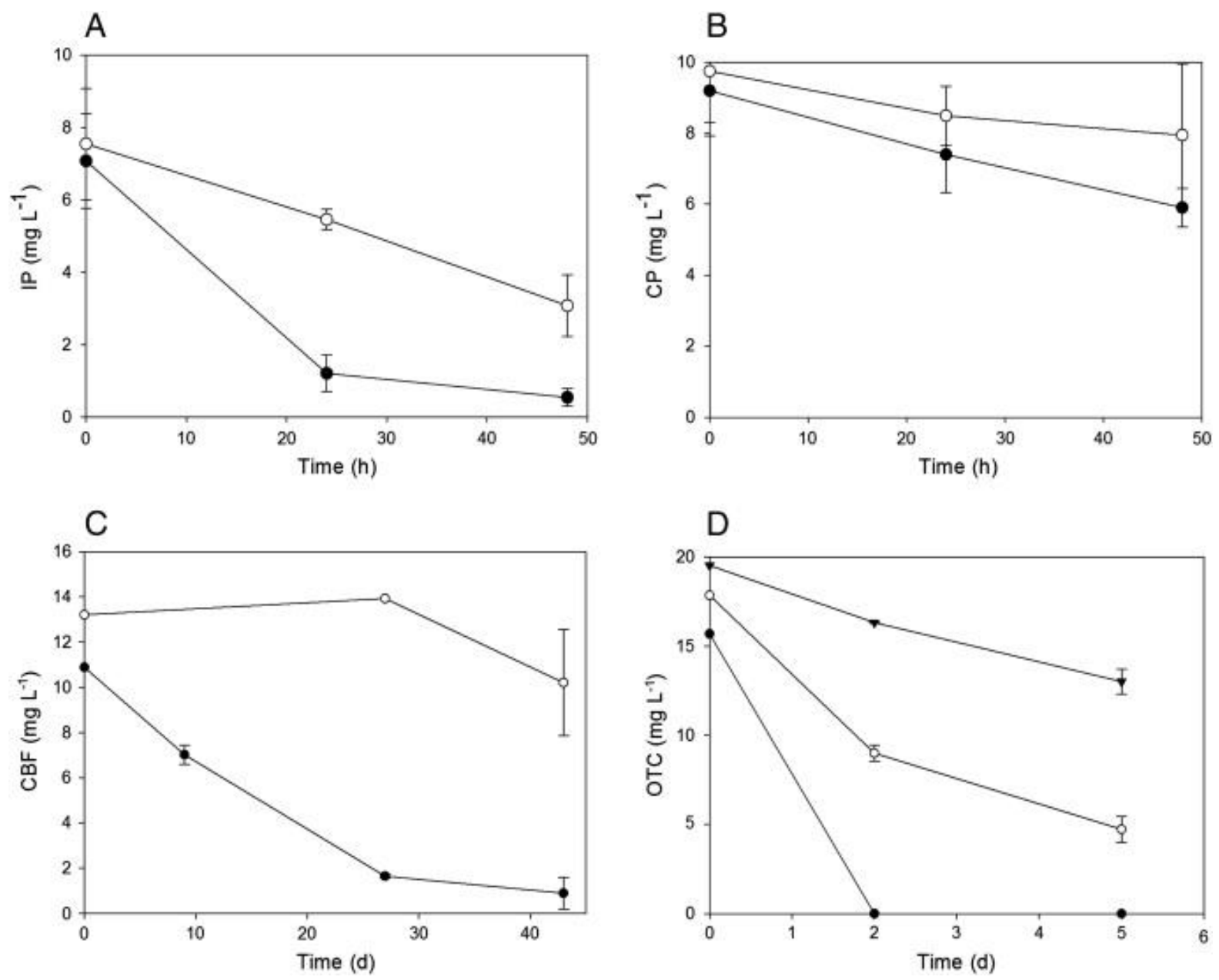

Fig. 4. Degradation profiles of IP (A), CP (B), CBF (C) and OTC (D) by T. versicolor in the presence of ABT as cytochrome P450 inhibitor. Sy mbols: inhibitor-free cultures $(\bullet)$, cultures supplemented with ABT $(\circ)$, abiotic $\operatorname{control}(\boldsymbol{\nabla})$. Values plotted are the means \pm SD for triplicate cultures.

Ecotoxicological evaluation was performed as most of the pyrethroids produce metabolites of higher toxicity than the parent compounds (Laffin et al., 2010). To assess the risk involved with the disposal of a potentially fungal-treated effluent containing $\mathrm{CP}$ and IP into the environment, a standard acute toxicity bioassay was performed with the bacterium $V$. fischeri. The initial pyrethroid concentration in the medium showed statistically the same $4 \mathrm{TU}$ (with a $95 \%$ of confidence) observed by the end of the process, probably due to the accumulation of similarly toxic transformation products.

\subsection{Degradation of CBF}

The degradation profile of $\mathrm{CBF}$ is shown in Fig. 1C. Abiotic losses and adsorption were negligible, according to control profiles. Although complete removal of the carbamate was not achieved, more than $96 \%$ was eliminated by the day 44 , with a half-live of around $22 \mathrm{~d}$, and an experimental degradation rate of $3.42 \mathrm{ng} \mathrm{h}^{-1} \mathrm{mg}^{-1} \mathrm{DCW}$ (dry cell weight). Removal of CBF is markedly slower than that of several organic pollutants tested with $T$. versicolor under similar conditions, including anti-inflammatories and antibiotics ( Marco-Urrea et al., 2009 and Prieto et al., 2011), and other agrochemicals 
from the present work; this observation can be ascribed mostly to the differences in the chemical structure of this more toxic pesticide compared to those organic contaminants. However the estimated half- life in this case was shorter than most values reported for dissipation of CBF in previously unexposed soils (Karpouzas et al., 2001).

Laccase activity was detected throughout the process and levels reached a maximum of $111 \mathrm{U} \mathrm{L}^{-1}$ by time $32 \mathrm{~d}$. However, assays conducted with purified laccase or laccase/mediators failed to reduce $\mathrm{CBF}$ concentration, thus indicating that this enzymatic complex is not involved in the transformation of the carbamate. Therefore, participation of cytochrome $\mathrm{P} 450$ was also evaluated. When the cytochrome $\mathrm{P} 450$ inhibitor ABT was added to the fungal cultures, a marked suppression of the removal was observed ( $0 \%$ elimination at time $27 \mathrm{~d}$ and $22 \%$ after $44 \mathrm{~d}$, respect $>87 \%$ in the free-inhibitor cultures, Fig. 4C). This finding strongly suggests that cytochrome P450 is involved in the transformation of CBF by $T$. versicolor.

The detection of one transformation product in the reaction cultures permitted to confirm that the removal of $\mathrm{CBF}$ was due to a degradation process. The profile of 3 hydroxycarbofuran production is shown in Fig. 5. This metabolite was detected for the first time after $9 \mathrm{~d}$, time after which its concentration continuously increased to reach a maximum concentration of $4.01 \mathrm{mg} \mathrm{L}^{-1}$ at time $38 \mathrm{~d}$. Only the final sampling point registered a slight decrease in concentration, when CBF was almost completely removed. 3-hydroxycarbofuran is regarded (alongside 3-ketocarbofuran) as one of the main CBF metabolites detected in diverse matrices, and shows more polarity but equally toxic effects on target and non-target organisms (Gupta, 1994). 3ketocarbofuran was also analyzed; however it was not detected in the fungal cultures. Fungal degradation of CBF has been reported with a Gliocladium sp strain, which used the pesticide as a sole carbon and energy source ( Slaoui et al., 2007), and with Mucor ramannianus, which in addition, produces the metabolite 3-hydroxycarbofuran-phenol from carbofuran-phenol ( Seo et al., 2007), analogous to the 3-hydroxycarbofuran detected in this work with T. versicolor.

Evaluation of the toxicity of the treatment revealed a reduction from $42 \mathrm{TU}$ at initial conditions to a residual 2 TU by time $22 \mathrm{~d}$ (half of the treatment) that was maintained at the end of the assay ( $3 \mathrm{TU})$, despite the accumulation of 3-hydroxycarbofuran.

\subsection{Degradation of OTC}

The removal of the antibiotic OTC is shown in Fig. 1D. Results obtained from abiotic and heat-killed controls ind icated that adsorption phenomena are negligible; similarly, these data also reveal high instability of the compound due to abiotic losses.

Nonetheless, comparison with reaction cultures showed a marked removal effect mediated by T. versicolor. That is, over $99 \%$ removal was achieved in the presence of viable biomass in $2 \mathrm{~d}$, respect $25 \%$ from abiotic losses in the same period. Dissipation rate from abiotic controls was estimated in $1.87 \mathrm{mg} \mathrm{L}^{-1} \mathrm{~d}^{-1}$, far below the $9.96 \mathrm{mg} \mathrm{L}^{-1}$ 
$\mathrm{d}^{-1}$ achieved with the fungus (equivalent to $65 \mathrm{ng} \mathrm{h}^{-1} \mathrm{mg}^{-1} \mathrm{DCW}$ ). This value is slightly lower compared to analogous assays performed with $T$. versicolor and other antibiotics such as sulfamethazine, sulfapyridine and sulfathiazole ( García-Galán et al., 2011, Rodríguez-Rodríguez et al., 2012a and Rodríguez-Rodríguez et al., 2012b). Results from this work showed a faster removal by $T$. versicolor than the WRF Pleurotus ostreatus, which required around two weeks for complete elimination of OTC ( Migliore et al., 2012).

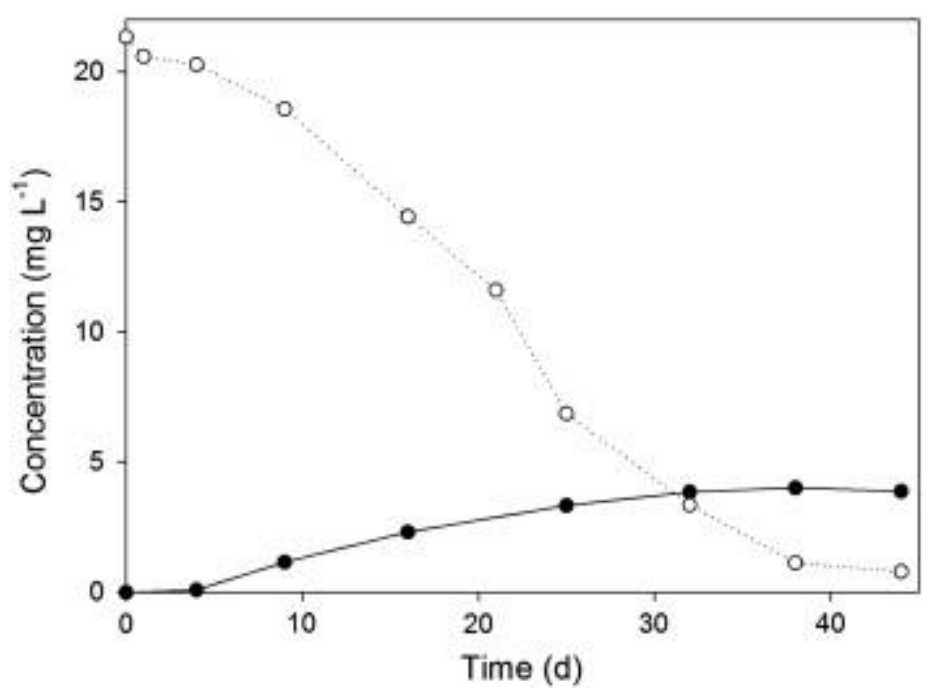

Fig. 5. Time-course evolution of 3-hydro xycarbofuran $(\bullet)$, major transformation product in the degradation of CBF (०) by T. versicolor pellets.

As for the case of CBF, laccase activity was detected during the whole period of the assay (maximum 156.7 $\mathrm{UA} \mathrm{L}^{-1}$ at day 3). Similarly, enzymatic experiments with purified laccase and laccase/mediator systems demonstrated that this enzyme is not involved in the early transformation of OTC, as the antibiotic concentration was not reduced in any case. Crude extracts of manganese peroxidase and lignin peroxidase from the WRF Phanerochaete chrysosporium successfully removed OTC (> 84\%) at levels of $50 \mathrm{mg} \mathrm{L}^{-1}$ in $4 \mathrm{~h}(84 \%)$ and $5 \mathrm{~min}$ (> 95\%) respectively ( Wen et al., 2009 and Wen et al., 2010); however activity of both enzymes is not significant in $T$. versicolor at work conditions. On the contrary, when ABT was employed as an inhibitor of cytochrome P450, assays with whole cells resulted in the drop of the removal rate of OTC (Fig. 4D). This finding suggests that the intracellular enzymatic complex is somehow involved in the transformation of OTC by T. versicolor.

Acute toxicity assays with $V$. fischeri showed an abrupt decrease in the toxicity of the medium, from $11 \mathrm{TU}$ at initial time to $3 \mathrm{TU}$ after $3 \mathrm{~d}$, which supports the environmental safety of the process.

Some reports portray the ability of $T$. versicolor to degrade pesticides other than those here studied. Previous works describe the increased removal of simazine, trifuralin and dieldrin when $T$. versicolor was added in wood chips to soil ( Fragoeiro and Magan, 2008); in the meantime, Karas et al. (2011) demonstrated the degradation of imazalil, 
thiophanate methyl, ortho-phenylphenol, diphenylamine and chlorpyrifos by the fungus in liquid media. Similarly, another species of the genus, Trametes hirsuta, has shown the ability to degrade endosulfan and endosulfan sulfate ( Kamei et al.,2011) and $\gamma$ hexchlorocyclohexane (lindane) (Singh and Kuhad, 1999), both in liquid media. Likewise, transformation of pesticides by laccases from $T$. versicolor and other WRF has been reported for the phenylurea $N^{\prime}, N^{\prime}$-(dimethyl)- $N$-(2-hydroxyphenyl)urea with immobilized enzyme ( Jolivalt et al, 2000) and bromoxynil, niclosamide, bromofenoxim and dichlorophen with a laccase-syringaldehyde system (Torres-Duarte et al., 2009). The versatility shown by $T$. versicolor strongly supports the potential of the fungus to remove a wide range of agrochemicals.

\subsection{Simultane ous degradation of agrochemicals}

Since CBF and OTC are similarly applied on soils, the degradation of a mixture of OTC and $\mathrm{CBF}$ was described using fungal biomass (Fig. 6A). Although a slight lag-phase was observed in CBF removal, the graphically estimated half-life for this compound was the same $(22 \mathrm{~d})$ as for cultures without OTC, and only $6 \%$ remained by the end of the treatment. The degradation rate was $3.47 \mathrm{ng} \mathrm{h}^{-1} \mathrm{mg}^{-1} \mathrm{DCW}$, practically the same observed without OTC. On the other hand, removal of OTC in the mixture was considerably delayed respect cultures without CBF (from 65 to $19.8 \mathrm{ng} \mathrm{h}^{-1} \mathrm{mg}^{-1}$ DCW); nonetheless, OTC removal was still faster than in the abiotic controls. Assays in the culture broth showed a gradual reduction in toxicity compared to the initial toxicity (20 TU); it decreased to 9 TU by day 21 and only to $1 \mathrm{TU}$ at the end of the experiment $(44 \mathrm{~d})$.

A second experiment with $\mathrm{CBF} / \mathrm{OTC}$ mixture was performed. This as say included successive additions of OTC during the second half of the treatment (Fig. 6 B). The amount of added OTC was systematically removed, including one spike of $40 \mathrm{mg} \mathrm{L}^{-1}$. As in the previous experiment, no apparent effect on the degradation of CBF was observed, and half-life was estimated in $21 \mathrm{~d}$ and degradation rate in $3.18 \mathrm{ng} \mathrm{h}^{-1} \mathrm{mg}^{-1}$ DCW. Although the last parameter seems slightly lower than in the other experiments, it was constant throughout the assay, and did not change after the successive OTC additions.

The simultaneous removal of CBF, OTC, CP and IP was similarly evaluated for a $21 \mathrm{~d}$ period (Fig. 7). No inhibition on fungal metabolism was observed, as indicated by glucose consumption profile and a peak in laccase activity $\left(37 \mathrm{U} \mathrm{L}^{-1}\right)$ similar to that obtained for this period in experiments solely with $\mathrm{CBF}$ or CBF/OTC. Moreover, the estimated half-life for CBF was $15 \mathrm{~d}$, shorter than other values obtained in this work. The profile of CP removal followed the same trend observed in the CP/IP mixture. In the meantime, delayed elimination was only detected for IP and OTC; however the former was completely removed by day 7 . Toxicological evaluation of the mixture showed a decrease in toxicity, from $10 \mathrm{TU}$ at time zero, to $4 \mathrm{TU}$ after $21 \mathrm{~d}$ of fungal treatment. 

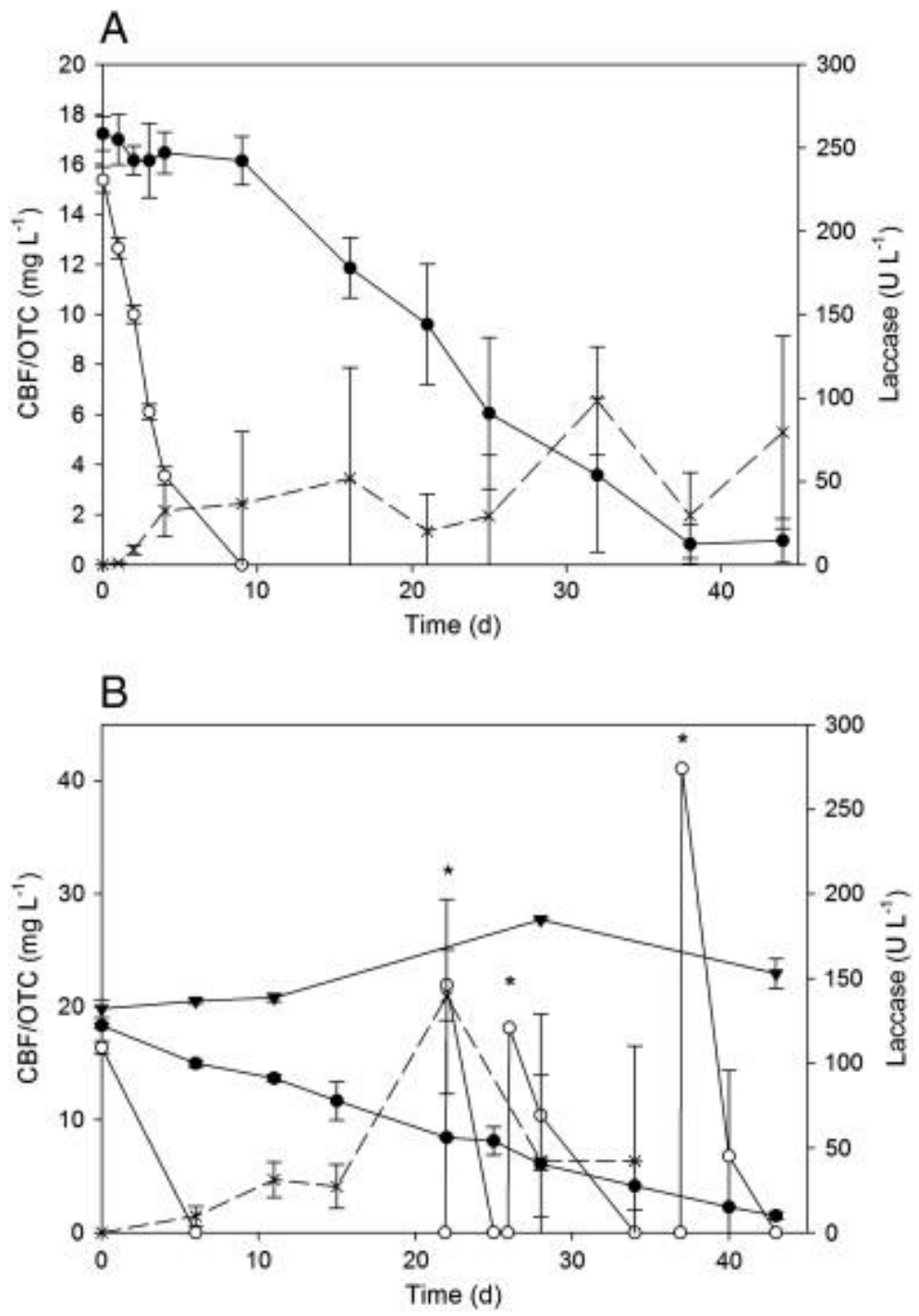

Fig. 6. Degradation profile of CBF/OTC mixtures: without successive additions of OTC (A); with successive additions of OTC (B). Sy mbols: CBF (•), OTC ( ०), CBF-abiotic control ( $\nabla)$, laccase activity $(\times)$. Time-points of OTC addition are marked with $(*)$. Values plotted are the means \pm SD for trip licate cultures.

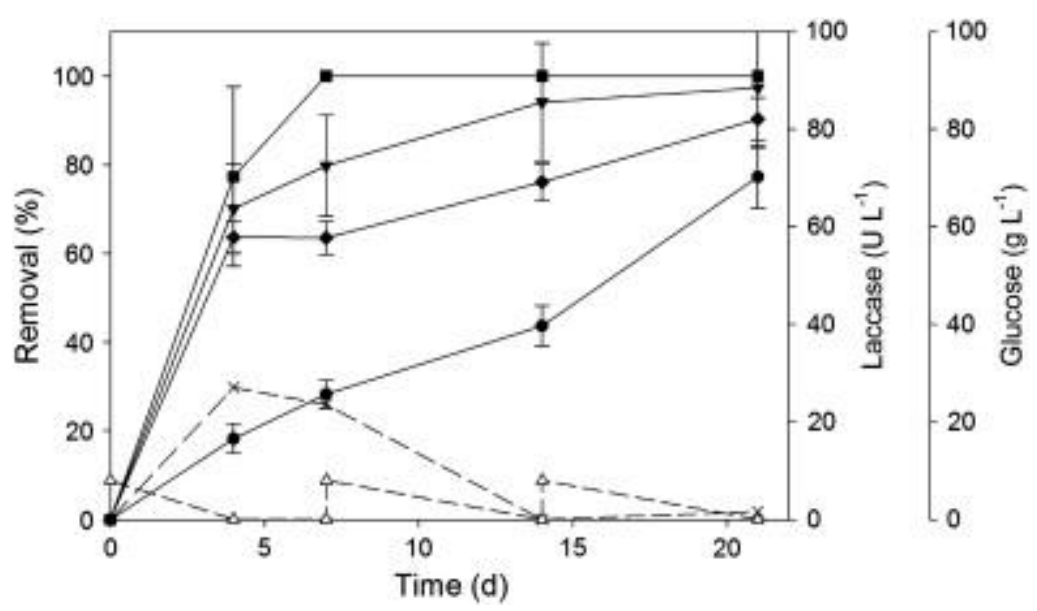

Fig. 7. Simultaneous degradation of IP $(\bullet), \mathrm{CP}(\bullet), \mathrm{CBF}(\bullet)$ and $\mathrm{OTC}(\boldsymbol{\nabla})$ by $T$. versicolor Glucose $(\Delta)$ and laccase activity $(\times)$ are also shown. Values plotted are the means \pm SD for triplicate cultures. 


\section{Conclusions}

Removal of diverse agrochemicals was achieved with $T$. versicolor. Degradation involved in the process was additionally confirmed in the cases of CBF and CP through the detection of transformation products. Simultaneous removal of the agrochemical mixtures supports not only the wide spectrum of oxidation capacity of the fungus, but also remarks its potential use to treat waste waters of agro- ind ustrial origin. Suitability of the process was evidenced by a reduction in the toxicity of the matrix after the fungal treatment. Slurry reactors and solid-phase biopile-like systems inoculated with $T$. versicolor have been successful in the removal of diverse organic emerging pollutants; similar configurations should be evaluated to assay their performance on the degradation of agrochemicals in the field.

\section{Acknowledgements}

This work was supported by the Spanish Ministries of MMAMRM (project 010/PC08/3-04) and MICINN (project CTQ2010-21776-C2-01), the Costa Rican Ministry of Science, Technology and Telecommunications MICITT (project FI-09313/802-B4-503), and Vicerrectoría de Investigación of Universidad de Costa Rica (project 802-B4-609). The Department of Chemical Engineering of the Universitat Autònoma de Barcelona is the Unit of Biochemical Engineering of the Xarxa de Referència en Biotecnologia de la Generalitat de Catalunya. C.E. Rodríguez-Rodríguez acknowledges UCR for a postdoctoral scholarship. 


\section{References}

Abdallah FB, Hamden K, Galeraud-Denis I, ElFeki A, Keskes-Ammar L. An in vitro study on reproductive toxicology of deltamethrin on rat spermatozoa. Andrologia 2010;42:254-9.

Aksakal E, Ceyhun SB, Erdoğan O, Ekinci D. Acute and long-term genotoxicity of delta- methrin to insulin-like growth factors and growth hormone in rainbow trout. Comp Biochem Physiol C 2010;152:451-5.

Asgher M, Bhatti HN, Ashraf M, Legge RL. Recent developments in biodegradation of in- dustrial pollutants by white rot fungi and their enzyme system. Biodegradation 2008;19:771-83.

Baiocco P, Barreca AM, Fabbrini M, Galli C, Gentili P. Promoting laccase activity towards non-phenolic substrates: a mechanistic investigation with some laccasemediator systems. Org Biomol Chem 2003;1:191-7.

Borchert M, Libra JA. Decolorization of reactive dyes by the white rot fungus Trametes versicolor in sequencing batch reactors. Biotechnol Bioeng 2001;75:313-21.

Cañas AI, Camarero S. Laccases and their natural mediators: biotechnological tools for sustainable eco-friendly processes. Biotechnol Adv 2010;28:694-705.

Canet R, Birnstingl JG, Malcolm DG, Lopez-Real JM, Beck AJ. Biodegradation of polycyclic aromatic hydrocarbons (PAHs) by native microflora and combinations of white-rot fungi in a coal-tar contaminated soil. Bioresour Technol 2001;76:113-7.

Chen S,Hu Q,HuM, Luo J,Weng Q, Lai K. Isolation and characterization of a fungus able to degrade pyrethroids and 3-phenoxybenzaldehyde. Bioresour Technol 2011;102: 8110-6.

Chen S, Luo J, Hu M, Geng P, Zhang Y. Microbial detoxification of bifenthrin by a novel yeast and its potential for contaminated soils treatment. PLoS One 2012;7:e30862.

Doddapaneni H, Yadav JS. Differential regulation and xenobiotic induction of tandem P450 monooxygenase genes pc-1 (CYP63A1) and pc-2 (CYP63 A2) in the white-rot fungus Phanerochaete chrysosporium. Appl Microbiol Biotechnol 2004;65:559-65.

Fabbrini M, Galli C, Gentili P. Comparing the catalytic efficiency of some mediators of laccase. J Mol Catal B Enzym 2002;16:231-40.

Font X, Caminal G, Gabarrell X, Romero S, VicentMT. Black liquor detoxification by laccase of Trametes versicolor pellets. J Chem Technol Biotechnol 2003;78:548-54. 
Fragoeiro S, Magan N. Impact of Trametes versicolor and Phanerochaete chrysosporium on differential breakdown of pesticidemixtures in soilmicrocosms at two water poten- tials and associated respiration and enzyme activity. Int Biodeter Biodegr 2008;62:376-83.

Gago-Ferrero P, Badia-Fabregat M, Olivares A, Piña B, Blánquez P, Vicent T, et al. Evalua- tion of fungal- and photo-degradation as potential treatments for the removal of sunscreens BP3 and BP1. Sci Total Environ 2012;427-428:355-63.

García-GalánMJ, Rodríguez-Rodríguez CE, Vicent T, Caminal G, Díaz-CruzMS, Barceló D. Biodegradation of sulfamethazine by Trametes versicolor: removal from sewage sludge and identification of intermediate products by UPLC-QqTOF. Sci Total Environ 2011;409:5505-12.

GuoP,Wang B,Hang B, Li L,Ali SW,He J, et al. Pyrethroid-degrading Sphingobiumsp. JZ-2 and the purification and characterization of a novel pyrethroid hydrolase. Int Biodeterior Biodegrad 2009;63:1107-12.

Gupta RC. Carbofuran toxicity. J Toxicol Environ Health 1994;43:383-418. Hata T, Kawai S, Okamura H, Nishida T. Removal of diclofenac andmefenamic acid by the white rot fungus Phanerochaete sordida YK-624 and identification of theirmetabolites after fungal transformation. Biodegradation 2010;21:681-9.

Hintzen EP, Lydy MJ, Belden JB. Occurrence and potential toxicity of pyrethroids and other insecticides in bed sediments of urban streams in central Texas. Environ Pollut 2009; 157:110-6.

Jolivalt C, Brenon S, Caminade E, Mougin C, Pontié M. Immobilization of laccase from Trametes versicolor on a modified PVDF microfiltration membrane: characterization of the grafted support and application in removing a phenylurea pesticide in waste- water. J Membrane Sci 2000;180:103-13.

Kamei I, Takagi K, Kondo R. Degradation of endosulfan and endosulfan sulfate by white- rot fungus Trametes hirsuta. JWood Sci 2011;57:317-22.

Karas P, Perruchon C, Exarhou K, Ehaliotis C, Karpouzas DG. Potential for bioremediation of agro-ind ustrial effluents with high loads of pesticides by selected fungi. Biodegra- dation 2011;22:215-28.

Karpouzas DG,Walker A, Drennan DSH, Froud-Williams RJ. The effect of initial concentra- tion of carbofuran on the development and stability of its enhanced biodegradation in top-soil and sub-soil. Pest Manag Sci 2001;57:72-81. 
Kirk TK, Schultz E, ConnorsWJ, Lorenz LF, Zeikus JG. Influence of culture parameters on lig- ninmetabolismby Phanerochaete chrysosporium. ArchMicrobiol 1978;117:27785 .

Kümmerer K. Resistance in the environment. J Antimicrob Chemother 2004;54:311-20. Kümmerer K. Antibiotics in the aquatic environment - Areview - Part 1. Chemosphere 2009;75:417-34.

Laffin B, Chavez M, Pine M. The pyrethroid metabolites 3-phenoxybenzoic acid and 3phenoxybenzyl alcohol do not exhibit estrogenic activity in the MCF-7 human breast carcinoma cell line or Sprague-Dawley rats. Toxicology 2010;267:39-44.

Lewis DFV, Wiseman A. A selective review of bacterial forms of cytochrome P450 enzymes. Enzym Microb Technol 2005;36:377-84.

Marco-Urrea E, Pérez-TrujilloM, Vicent T, Caminal G. Ability ofwhite-rot fungi to remove selected pharmaceuticals and identification of degradation products of ibuprofen by Trametes versicolor. Chemosphere 2009;74:765-72.

Mendoza-Cantu A, Albores A, Fernandez-Linares L, Rodriguez-Vasquez R. Pentachloro- phenolbiodegradation and detoxification by the white-rot fungus Phanerochaete chrysosporium. Environ Toxicol 2000;15:107-13.

Migliore L, Fiori M, Spadoni A, Galli E. Biodegradation of oxytetracycline by Pleurotus ostreatus mycelium: a mycoremediation technique. J Hazard Mater 2012;215216: $227-32$.

Olsson AO, Baker SE, Nguyen JV, Lomanoff LC, Udunka SO, Walker RD, et al. A liquid chromatography-tandemmass spectrometrymultiresiduemethod for quantification of specific metabolites of organophosphorous pesticides, synthetic pyrethroids, selected herbicides, and DEET in human urine. Anal Che m 2004;76:2453-61.

Plangk lang P, Reungsang A. Bioaugmentation of carbofuran by Burkholderia cepacia PCL3 in a bioslurry phase sequencing batch reactor. Process Biochem 2010;45:230-8.

Prieto A, Möder M, Rodil R, Adrian L, Marco-Urrea E. Degradation of the antibiotics norfloxacin and ciprofloxacin by awhite-rot fungus and identification of degradation products. Bioresour Technol 2011;102:10987-95.

Rodríguez-Rodríguez CE, Barón E, Gago-Ferrero P, Jelić A, LlorcaM, FarréM, et al. Removal of pharmaceuticals, polybrominated flame retardants and UV-filters from sludge by the fungus Trametes versicolor in bioslurry reactor. J Hazard Mater 2012a;233-234:235-43. 
Rodríguez-Rodríguez CE, García-Galán MJ, Blánquez P, Díaz-Cruz MS, Barceló D, Caminal G, et al. Continuous degradation of a mixture of sulfonamides by Trametes versicolor and identification of metabolites from sulfapyridine and sulfathiazole. $\mathrm{J}$ Hazard Mater 2012b;213-214:347-54.

Seo J,JeonJ,Kim SD,KangS,Han J,HurHG. Fungal degradation of carbofuran and carbofuranphenol by the fungus Mucor ramannianus: identification of metabolites. Water Sci Technol 2007;55:163-7.

Sequinatto L. Occurrence of agrochemicals in surface waters of shallow soils and steep slopes cropped to tobacco. Quim Nova 2013;36:768-72.

Shafer TJ,Meyer DA, CroftonKM. Developmental neurotoxicity of pyrethroid insecticides: critical review and future research needs. Environ Health Perspect 2005;113:123-36.

Singh BK, Kuhad RC. Biodegradation of lindane ( $\gamma$-hexachlorocyclohexane) by thewhite- rot fungus Trametes hirsutus. Lett Appl Microbiol 1999;28:238-41.

Slaoui M, Ouhssine M, Berny E, Elyachioui M. Biodegradation of carbofuran by a fungus isolated from treated soil. Afr J Biotechnol 2007;6:419-23.

Swamy J, Ramsay JA. The evaluation ofwhite rot fungi in the decoloration of textile dyes. Enzym Microb Technol 1999;24:130-7.

Torres-Duarte C, Roman R, Tinoco R, Vazquez-Duhalt R. Halogenated pesticide transfor- mation by a laccase-mediator system. Chemosphere 2009;77:687-92.

Vidaver A. Uses of antimicrobials in plant agriculture. Clin Infect Dis 2002;34(Suppl. 3): S107-10.

Wang B, Ma Y, ZhouW, Zheng J, Zhu J, He J, et al. Biodegradation of synthetic pyrethroids by Ochrobactrum tritici strain pyd-1.World JMicrobiol Biotechnol 2011;27:2315-24.

Wariishi H. Manganese (II) oxidation by manganese peroxidase from the basidiomycete Phanerochaete chrysosporium. J Biol Chem 1992;267:23688-95.

Wen X, Jia Y, Li J. Degradation of tetracycline and oxytetracycline by crude lignin peroxi- dase prepared fromPhanerochaete chrysosporium-awhite rot fungus.

Chemosphere 2009;75:1003-7.

Wen X, Jia Y, Li J. Enzymatic degradation of tetracycline and oxytetracycline by crude manganese peroxidase prepared from Phanerochaete chrysosporium. J Hazard Mater 2010;177:924-8. 
Yuan F, Hu C, Hu X, Wei D, Chen Y, Qu J. Photodegradation and toxicity changes of anti- biotics in UV and UV/H2O2 process. J Hazard Mater 2011;185:1256-63.

Zhang C, Jia L,Wang SH,Qu J,Xu LL, ShiHH, et al. Biodegradation of betacypermethrin by two Serratia spp. with different cell surface hydrophobicity. Bioresour Technol 2010; 101:3423-9.

ZhengZM, Obbard JP. Effect of non-ionic surfactants on elimination of polycyclic aromatic hydrocarbons (PAHs) in soil-slurry by Phanerochaete chrysosporium. J Chem Technol Biotechnol 2001;76:423-9.

Zhou J, JiangWY, Ding JA, Zhang XD, Gao SX. Effect of Tween 80 and betacyclodextrin on degradation of decabromodiphenyl ether (BDE-209) bywhite rot fungi. Chemosphere 2007;70:172-7. 